\title{
COHERENT ELECTRON BEAM DENSITY MODULATOR FOR DRIVING X-RAY FREE ELECTRON LASERS
}

\author{
A. Novokhatski\#, F.-J. Decker, B. Hettel, Yu. Nosochkov, M. Sullivan \\ SLAC National Accelerator Laboratory, Menlo Park, CA, USA
}

\begin{abstract}
We propose a new compact scheme for a Free Electron Laser with more coherent properties for the X-ray beam. Higher FEL performance would be achieved using a train of electron bunches initially accelerated in a linear accelerator. Similar to the RF klystron concept, we propose developing an X-ray FEL which consists of two parts: an Xray self-seeding electron beam density modulator and an output set of undulators. A density modulator consists of a low-Q X-ray cavity and an undulator, which is placed between the cavity mirrors. We use this undulator as a very high gain amplifier, which compensates the amplitude loss due to monochromatic X-ray reflections from the mirrors. Following the X-ray cavity, the density modulated electron beam is separated from the X-ray beam and then enters the output set of undulators. The frequency spectrum of the final X-ray beam is determined mainly by the bandwidth of the reflected elements in the X-ray cavity.
\end{abstract}

\section{INTRODUCTION}

The success of the hard X-ray self-seeding experiment (HXRSS) [1] at the LCLS is very important in that it provides a narrow, nearly transform-limited bandwidth from the FEL, fulfilling a beam quality requirement for experimental applications requiring highly monochromatic X-rays. Self-seeding a FEL using an X-ray beam generated in the first half of the undulator to seed the second half for soft and hard X-rays was first proposed in 1997 [2]. More details about the hard X-ray sel-seeding scheme are given in [3]. Yet, because the HXRSS signal is generated from random spikes of noise, the seeded beam suffers from large fluctuations in intensity due to the random nature of generation. This is exacerbated by any electron beam energy jitter. In addition, in the HXRSS scheme, the electron beam gets a coherent energy spread while generating an X-ray beam in the first part of the undulator. This additional energy spread is smeared in the HXRSS chicane, and this effectively increases the energy spread of the beam entering in the second part of the undulator.

This proposal is similar to the RF klystron concept (a klystron has an input cavity, several "buncher" cavities and an output cavity). We propose developing an X-ray FEL which consists of two parts: an X-ray self-seeding electron beam energy and density modulator and an output set of undulators, where the beam produces high power X-ray beams. We use a train of electron bunches initially accelerated in a linear accelerator. Each bunch in a train (except the first one) is a "fresh" bunch meaning that it keeps properties gained in the gun and the linac. It interacts with the X-ray fields, produced by the previous bunches. The electron beam density modulator in our proposal consists of a low-Q X-ray cavity and an undulator, which is placed between the cavity mirrors. The cavity acts as a return path for the fields, produced in this undulator.

We would like to mention that a common approach for the near infrared free electron laser master oscillator/power amplifier (FEL-MOPA) was discussed before in $[4,5,6]$. However we do not assume that the hard X-ray fields (which have much higher frequencies) can be coherently stored in the cavity as also proposed in XFELO [7], and therefore we do not need tight tolerances on the cavity length in this proposal. We assume that instead the undulator inside the X-ray cavity works as a very high gain amplifier, which compensates the amplitude loss due to the monochromatic X-ray reflections from the mirrors.

The low-Q cavity consists of several mirrors, which can reflect X-rays by several degrees to make a total of 360 degrees. These mirrors could be four crystals with a corresponding Bragg angle of about 45 degrees each with additional small angle correcting elements. In this case, the amplitude loss is due to the small bandwidth of the reflected fields. After the X-ray cavity, the density modulated electron beam is separated from the X-ray beam by a chicane and then enters the output set of undulators. The modulated electron beam then produces a monochromatic X-ray beam in these undulators. The frequency spectrum of the final X-ray beam is determined mainly by the density modulation of the electron beam and not by SASE performance. Only a few undulators would be needed to reach saturation. The bandwidth of the X-ray beam is mainly determined by the bandwidth of the reflection coefficients of the reflecting elements (crystals) in the X-ray cavity. 
In this study, we analyze the interaction of the X-rays and the electron beam. The electron beam source in this proposal is a train of electron bunches initially accelerated in a linear accelerator. This beam then passes through a radiator element situated within an X-ray cavity which modulates the beam. After modulation the beam goes through an output set of undulators for final generation of monochromatic X-ray beams. Our concept for the electron beam density modulator is very similar to a regenerative-amplifier FEL (RAFEL), which has been demonstrated in the infrared wavelength region [8]. It was also proposed for the 4th Generation Light Source (VUV-FEL) at Daresbury Laboratory [9] and discussed in the angstrom wavelength region [10]. However our concept for the hard X-ray beam is different. It will be less sensitive to the electron beam energy jitter and have less unavoidable SASE radiation. A preliminary version of this concept was presented in a conference paper [11], but here our design is more detailed and much more complete. Our concept should also work very well for soft X-ray beams. In this case, the design would also be more compact.

\section{A CONCEPT}

The basic schematic is shown in Fig. 1. The electron beam is accelerated in a SLAC-type linac or a superconducting linac. It then enters an electron beam density modulator or, in other words, an X-ray cavity with undulators. We may use several undulators of the LCLS type [12] as the radiator element inside the X-ray cavity. The same type of undulators can be used in the output set. For the X-ray mirrors, we propose the same type of crystals that are currently in use in the XCS experiment (The X-ray Split Pulse Experiment) at the LCLS [13]. In this case, there are two chicanes which provide a path for the electron beam around the X-ray cavity mirrors. The electron beam goes through the first chicane avoiding the X-ray cavity mirror, then passes through the cavity undulator making the X-ray beam for the cavity, and then goes through the second chicane again avoiding the X-ray cavity mirror. Finally, it enters the output set of undulators. SASE radiation from the leading electron bunch in a bunch train is spectrally filtered by the Bragg crystalreflectors and is brought back to the beginning of the cavity undulator to interact with the second beam bunch. The Xray pulse that circulates in the cavity repeatedly interacts with consecutive electron bunches in the train, forming a regenerative amplifier FEL. This process yields a growing laser field in the X-ray cavity if the amplification of the field in the cavity undulator is more than the reflection losses. In this case, the final amplitude of the X-ray field will be limited by non-linear effects of the electron beam interacting with the X-ray beam. Naturally, heating effects in the mirrors (crystals) must be taken into consideration. The FEL interaction with these short bunches regeneratively amplifies the radiation intensity because the crystal reflectors filter the radiation, making the frequency bandwidth smaller. The last bunch of a train (or all previous bunches), after getting perfect density modulation, goes into the output set of the undulators and produces high power monochromatic radiation. The charge of the last bunch also can be higher than in the previous bunches in order to get more FEL power. Compared to a SASE X-ray FEL, this approach should need a shorter output undulator length. A small number of electron bunches may generate multi-GW X-ray pulses with excellent temporal coherence. The resulting spectral brightness of these X-ray pulses can be another 2 to 3 orders of magnitude higher. It is important to mention that we do not use the X-ray cavity in a fundamental way, as a cavity with resonator eigenmodes. We use only the last return X-ray pulse, which modulates the next coming bunch. Due to the large single-pass gain in the X-ray cavity, the output intensity at the cavity exit is orders of magnitude above the input.

As with a classical FEL, the beam energy (a few $\mathrm{GeV}$ ) corresponds to the radiation wavelength. The beam energy spread and beam emittance must not be above the usual FEL requirement. The electron bunch pattern may consist of an initial train of relatively low current bunches followed by a high current bunch. The bunch spacing depends upon the double length of the distance between the reflecting crystal-mirrors. However there is no strong requirement on the arrival time because the reflected X-ray pulse length is increased $(\sim \mathrm{ps})$ due to the frequency filtering (because of the multiple reflections inside the atomic layers of the crystal).

To demonstrate the advantage of our concept relative to an X-ray FEL of the LCLS type [12], we performed comparison simulations.

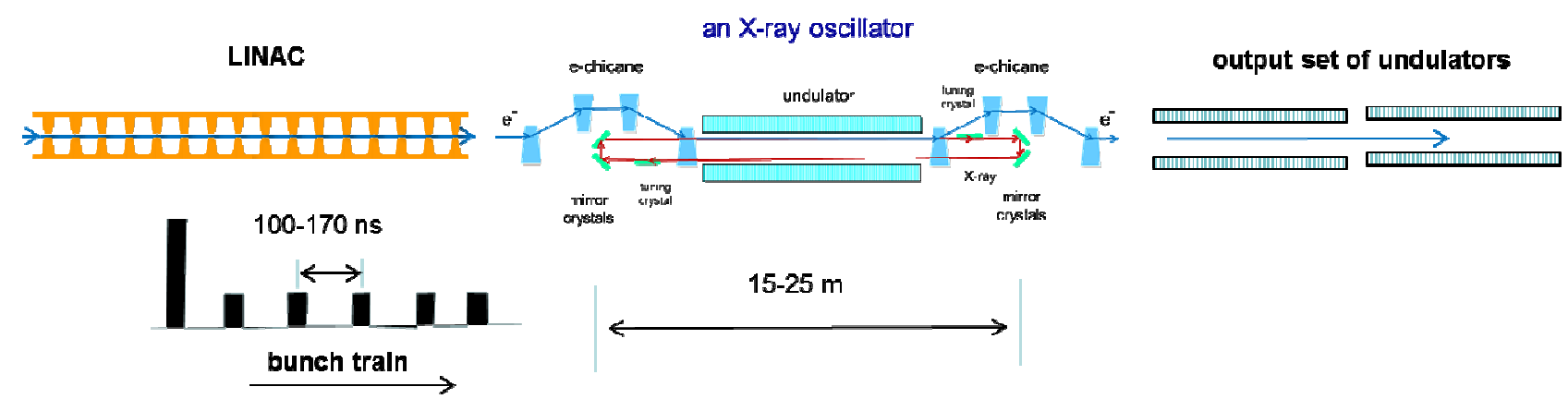

Figure 1: A proposed layout of an FEL using an X-ray oscillator as an electron beam density modulator. 


\section{SIMULATION METHOD}

We have simulated the proposed FEL scheme using numerical solutions of the particle and X-ray field equations in the one-dimensional FEL approach $[14,15]$. This was performed using a numerical code developed by

Z. Huang [16], which we modified for our case. The modifications include a different kind of filtering method for the $\mathrm{X}$-ray field due to Bragg reflection, and we also take into account the motion of the electron beam in the chicanes. We use a convolution method to calculate the reflected X-ray signal:

$$
E(t)=e^{-i \omega t} \int_{-\infty}^{\infty} E\left(t-\tau-\tau_{p h}\right) W\left(\tau, \sigma_{\omega}\right) d \tau
$$

together with a Gaussian filter:

$W\left(\tau, \sigma_{\omega}\right)=\frac{\sigma_{\omega}}{\sqrt{2 \pi}} e^{-\frac{\left(\sigma_{\omega} \tau\right)^{2}}{2}}$

or a rectangular filter:

$W\left(\tau, \sigma_{\omega}\right)=\frac{\sin \left(\frac{\sigma_{\omega} \tau}{2}\right)}{\pi \frac{\tau}{2}}$

where $\Delta \omega$ is the difference between a frequency of the undulator radiation and the central frequency of the filter. The chicane is described by a $R 56$ parameter. After the chicane, a particle phase $\varphi_{i}$ changes according to its relative energy $\Delta \gamma_{i}$

$\varphi_{i}=\varphi_{i}+\frac{2 \pi}{\lambda} R_{56} \frac{\Delta \gamma_{i}}{\gamma}$

We use a code "XOP" [17] to calculate the crystal mirrors reflecting parameters. We also calculate the phase of the reflected signal using the Kramers - Kronig relations [18, 19], based on the real part of the reflected signal. The phase of the reflected signal is generally linear inside the main gap of reflections and is considered to be a time shift $\tau_{p h}$ in the time-domain - see equation (1).

\section{ESTIMATES OF THE FEL POWER AND BANDWIDTH IN SASE OPERATION}

In the simulations, we take approximately the same parameters for the undulators and the beam as they are in SASE operation of the LCLS. We consider the X-ray wavelength to be around $0.15 \mathrm{~nm}$, the FEL beam parameter to be $5 \cdot 10^{-4}$ and the total undulator length of $56 \mathrm{~m}$. An electron bunch has a rectangular shape. The bunch length is chosen to be $16.5 \mu \mathrm{m}$ (or $55 \mathrm{fs}$ ) in order to have a frequency resolution better than $1 \cdot 10^{-5}$. The bunch charge is $150 \mathrm{pC}$ and the bunch current is $3 \mathrm{kA}$. In SASE operation at the LCLS, these parameters provide the following results: output averaged power of $(1.5-2.0) \cdot 10^{10} \mathrm{~W}$, the relative R.M.S. bandwidth of $(5-6) \cdot 10^{-4}$ and the pulse energy of $1 \mathrm{~mJ}$.

The detailed results of computer simulations are shown in the following plots: the power gain along the undulators (Fig. 2), the time structure of the X-ray pulse (Fig. 3) and its frequency spectrum (Fig. 4). These simulation results are very close to the experimental measurements at LCLS without undulator tapering. 


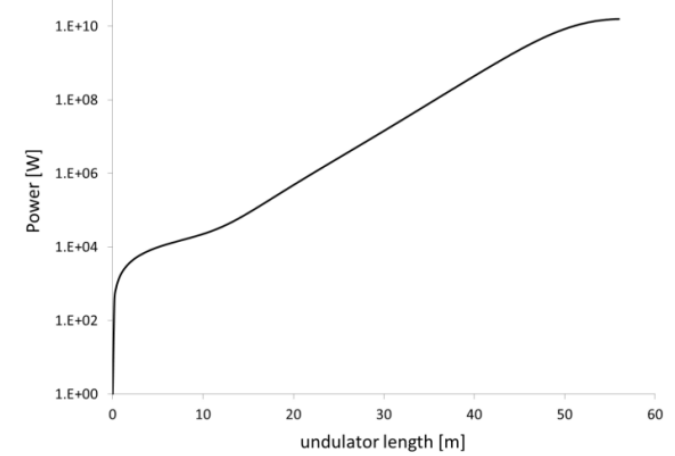

Figure 2: Power gain along the undulators.

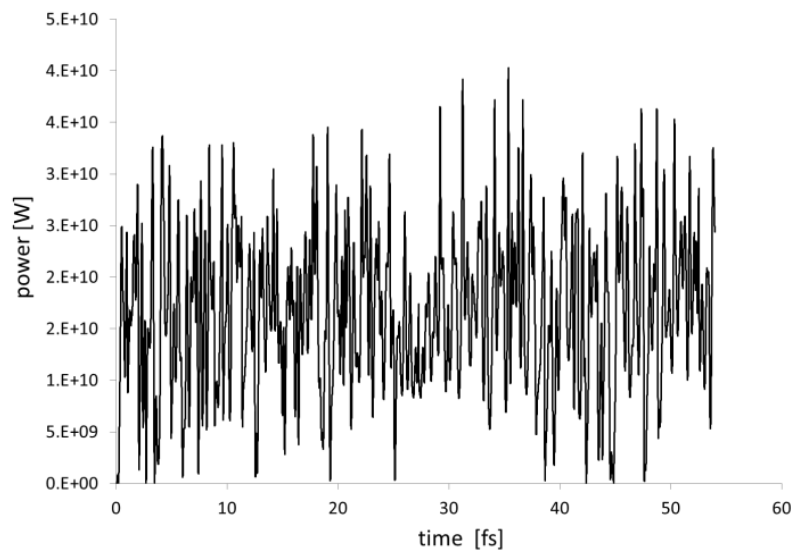

Figure 3: Time structure of the radiation power.

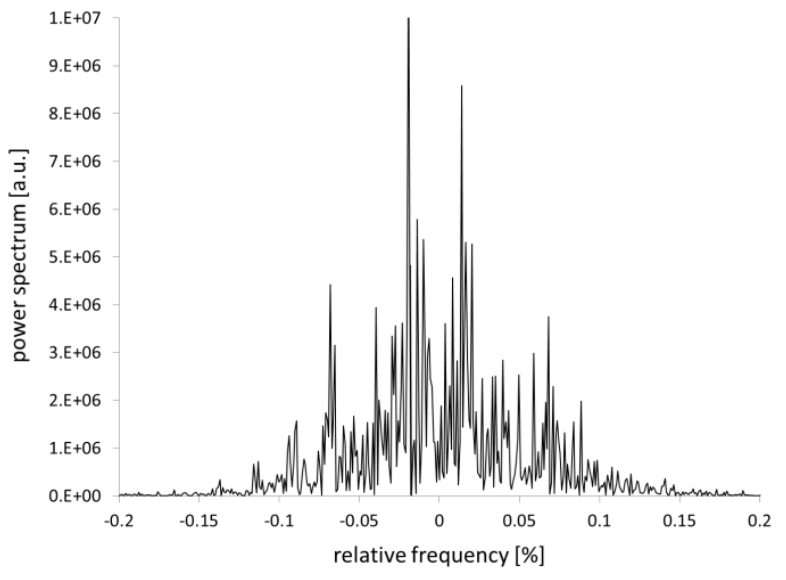

Figure 4: Spectrum of the output signal.

\section{REFLECTION LOSSES FROM MIRROR CRYSTALS}

The use of crystals as an X-ray mirror brings frequency filtering, that in some sense means power loss, not only for the out-band frequencies but also for the main frequency due to lengthening of the pulse. To estimate the power of the Xray beam signal after the reflections from crystals, we use a Gaussian filter (2) and the initial signal with approximately the same time structure as in the output signal from the undulators. We filtered for various amplitudes and then calculated the average power. The power transmission after reflections from four crystals is shown in Fig. 5 as a function of the relative bandwidth of the filter. As one can see in Fig. 5, to make an X-ray beam bandwidth less than $10^{-5}$, we need to recover a power loss of 20-30 dB in order to make the reflected X-ray signal dominating the fields due to the stochastic beam structure. To do this, according to Fig. 2, we need at least 2-3 SLAC-type undulators (8-12 m). 


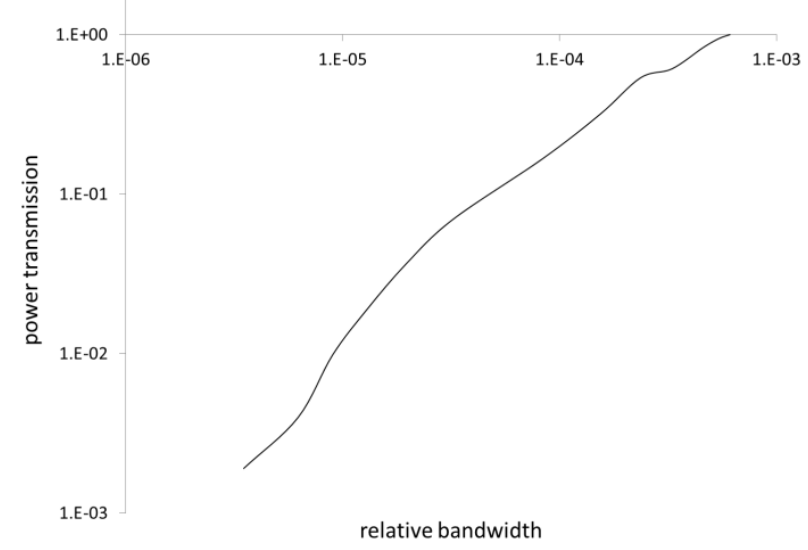

Figure 5: Power transmission as a function of the reflection filtering.

We use the code "XOP" in order to help us identifying the type of crystal and its parameters for good reflectivity in the range of Hard X-rays $(\sim 8 \mathrm{keV})$. For an X-ray cavity that is made of four mirrors, the reflection angle must therefore be 45 degrees. We found that a $100 \mu \mathrm{m}$ thick diamond crystal with Miller indices of (311) provides good reflection at a Bragg angle of 45 degrees and a wavelength of $0.152 \mathrm{~nm}$. The reflectivity curve of p-polarized radiation from four crystals is shown in Fig. 6. The reflectivity shape is not very far from rectangular, having a small (10\%) slope. Transmission power is not high, but enough to make the Q-value of X-cavity itself less than 10 . The total bandwidth is around $1.0 \cdot 10^{-5}$ and the R.M.S. bandwidth is $3.0 \cdot 10^{-6}$.

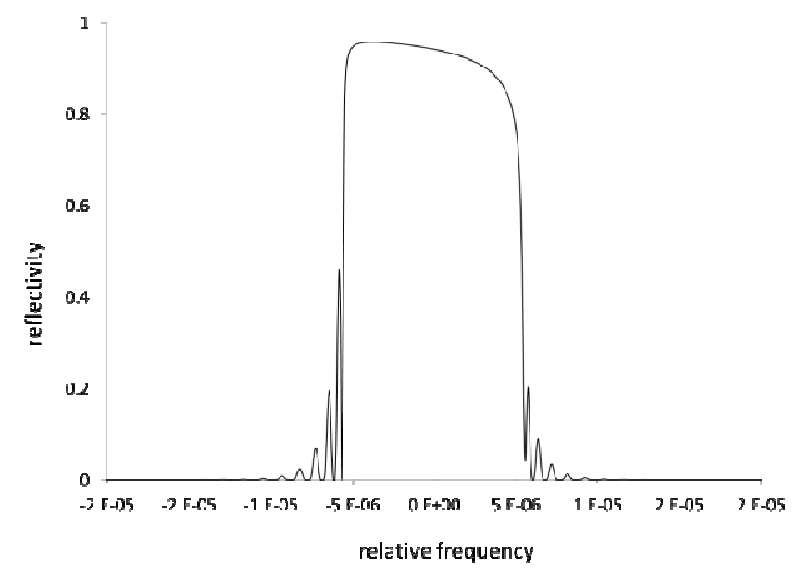

Figure 6: Reflectivity spectrum of four diamond crystals.

\section{SIMULATIONS OF THE BEAM DENSITY MODULATOR}

In order to perform simulation of the proposed scheme, first, we check how many undulators are needed inside the Xray cavity. Results of calculations of the X-ray beam power in the X-ray cavity for different numbers of undulators and a train of several electron bunches show that the minimum number of undulators inside the X-ray cavity must be more than three $(12 \mathrm{~m})$. In this case, the amplification of the monochromatic signal compensates the reflection losses in the mirrors. This number confirms our previous estimate. According to our proposal, after the X-ray cavity, we then use only a density modulated electron beam to produce the final X-ray beam power. So, this power depends upon the level of density modulation and the number of undulators after the X-ray cavity. We found that if we keep the same total length of undulators (as we use for SASE simulation) including undulators inside the X-ray cavity, we will need a train of only 3 electron bunches to come to saturation in the last undulator. In this case, we will achieve the same output power, but with a smaller bandwidth compared to the "classical" SASE LCLS operation. If we continue to increase the number of bunches, we can get a much smaller bandwidth for a shorter total undulator length (fewer undulators after the $\mathrm{X}$-ray cavity). The number of bunches, the total undulator length, the output power and the bandwidth are shown in Table 1.

We may consider the case of a train of 6 electron bunches, because the total electron beam pulse will correspond to the RF pulse length of the SLAC linac. In this case, our proposed scheme promises the same power X-ray beam as in SASE operation, but with a bandwidth that is ten times smaller and this works with $30 \%$ fewer undulators. 
Table 1: Achievable bandwidth

\begin{tabular}{lllll}
\hline $\begin{array}{l}\text { Number of } \\
\text { bunches }\end{array}$ & $\begin{array}{l}\text { Total pulse } \\
\text { length }[\boldsymbol{\mu s}]\end{array}$ & $\begin{array}{l}\text { Undulator } \\
\text { length }[\mathrm{m}]\end{array}$ & $\begin{array}{l}\text { Output } \\
\text { power }[\mathrm{W}]\end{array}$ & Bandwidth \\
\hline 1 (LCLS) & - & 56 & $1.63 \cdot 10^{10}$ & $5.2 \cdot 10^{-4}$ \\
\hline 3 & 0.3 & 56 & $1.66 \cdot 10^{10}$ & $3.9 \cdot 10^{-4}$ \\
\hline 6 & 0.6 & 40 & $1.74 \cdot 10^{10}$ & $4.3 \cdot 10^{-5}$ \\
\hline 8 & 0.8 & 28 & $1.77 \cdot 10^{10}$ & $1.6 \cdot 10^{-5}$ \\
\hline
\end{tabular}

To see the difference with the SASE operation, presented in Fig. 2-4, we show plots of the same parameters, achieved in our proposal. Fig. 7 shows the power gain in undulators including X-ray cavity undulators for the case of 6 bunches and $40 \mathrm{~m}$ of the undulators. This power gain corresponds to the last (6th) electron bunch. One may compare this plot with a plot shown in Fig. 2. The time signal or power distribution along the last bunch is shown in Fig. 8 . The distribution becomes much smoother in comparison to the SASE operation (Fig. 3).

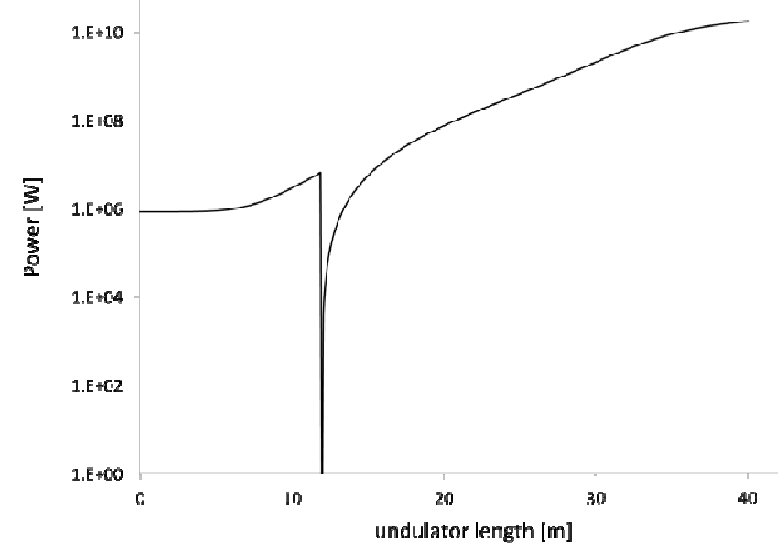

Figure 7: Power gain in the X-ray cavity versus the output set of undulators.

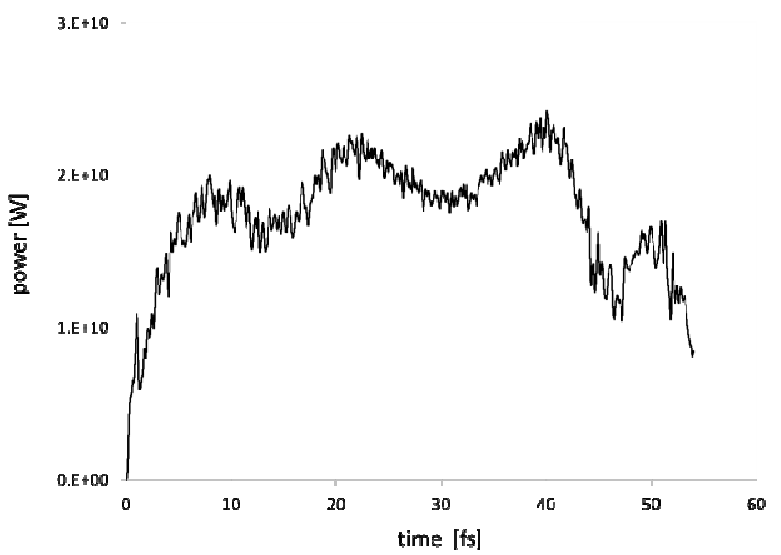

to the last electron bunch.

Figure 8: Time structure of the radiation power corresponding

The spectrum of the X-ray beam also becomes much more monochromatic. Fig. 9 shows the spectrum of the X-ray beam, which corresponds to the last electron bunch. To make a comparison with the spectrum during regular SASE operation (Fig. 4), we need to take into account that the frequency scale is ten times smaller (Fig. 9). We can see also that the amplitude is ten times larger. 


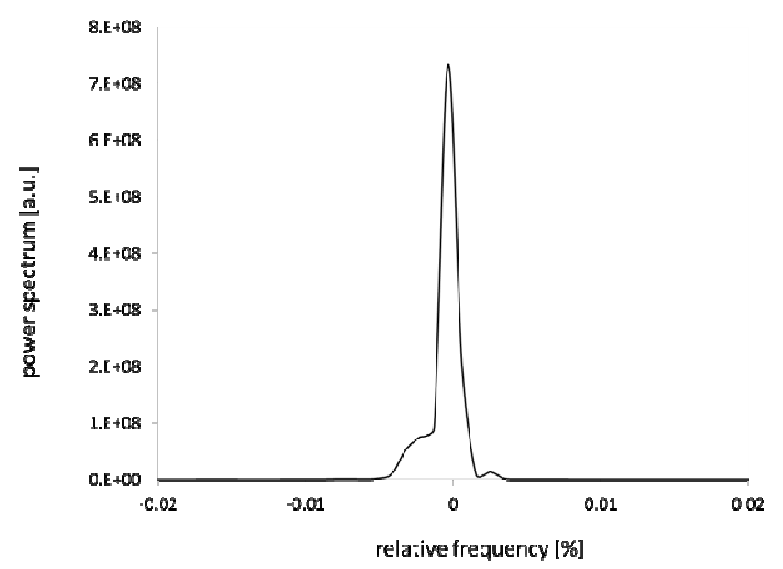

Figure 9: Spectrum of the output signal. The frequency scale is ten times smaller than the scale at Fig.4.

\section{THE DOWNSTREAM CHICANE}

In our proposal, we use two chicanes which provide a path for the electron beam around the X-ray cavity mirrors. The first chicane does not have much influence on the radiation process; however the second chicane may have a very strong effect. Depending upon the momentum compaction parameter ( $R 56)$ the electron beam density modulation can be increased or completely smoothed out. To estimate how small R56 must be, we may suggest that the particles must not be allowed to change their positions more than a half of the wavelength while passing through the chicane. Under this condition and using relation (4), we can get an estimate

$$
\left|R_{56}\right| \ll \frac{\lambda}{2} / \frac{\Delta \gamma_{i}}{\gamma}
$$

In most FEL projects the relative energy spread is usually of order of $10^{-4}$, then for a wavelength of $0.15 \mathrm{~nm}$ the absolute value of the momentum compaction (R56) must be less than one micron. Can we make a chicane with such a small R56 ? The answer is "yes", because we do not need a large crystal; a $2 \mathrm{~mm}$ size will be enough, and the length between magnets in the chicane may be of order of several meters. A quick estimate for a symmetric 4-bend chicane gives a formula from the reference [20]

$$
R_{56} \approx-2 \frac{h^{2}}{L}
$$

where $h$ is the displacement near the crystal situated in the middle of the chicane, and $L$ is the distance between the centers of the first and the second magnets. The same distance is between the third and the fourth magnets. We can get $R 56=-1 \mu \mathrm{m}$ for the $h=2 \mathrm{~mm}$ and $L=8 \mathrm{~m}$. More precise calculations using MAD [21] confirmed this estimate.

In the previous simulations of our proposed scheme the momentum compaction parameter was zero. Now we include it in the simulations and find confirmation of our estimate (5) of the maximum $\mid$ R56l. Fig. 10 shows distributions of the particles on the phase plane of energy and coordinate for $R_{56}=0$ (Fig. 10a) and R56=-2 $\mu \mathrm{m}$ (Fig. 10b) at the end of the last undulator. We can see clearly the over-compression of the micro bunch at the $-2 \mu \mathrm{m}$ momentum compaction. This effect diminishes the main harmonic beam current, which leads to smaller radiation power and more than doubles the bandwidth of the X-ray beam. However, at this momentum compaction we can still get a good result if we decrease the length of the undulators after the X-ray cavity from $28 \mathrm{~m}$ to $23 \mathrm{~m}$. The particle distribution on the phase plane for this case is shown in Fig. 10c which shows that the momentum compaction of $-2 \mu \mathrm{m}$ helps to optimize the installation. Therefore, the distance between the magnets in the chicane can be half as long $(4 \mathrm{~m})$, and the total length of the undulators $(\mathrm{Lu})$ can be reduced to $35 \mathrm{~m}$. We did not find any better results for larger values of the $|R 56|$. As a next step, we plan to analyze the beam dynamics more accurately using $3 \mathrm{D}$ calculations. However, it is interesting to note that a chicane with an extremely low R56 already exists at the LCLS and is used in operation when HXRSS is not used. In this case, an electron beam goes almost straight over a distance equal to the length of one undulator unit ( $\sim \mathrm{m})$. There are additional coils at all four chicane magnets, which are used to make a small deflection of the beam in order to control the delay of the beam versus the X-ray beam. This chicane works over a wide range of beam parameters without 
disturbing the electron beam quality. In Fig. 11 we show a possible scheme of the chicane lattice. Our chicane is longer than the LCLS HXRSS chicane, and we will use the 3D calculations to check the beam stability.

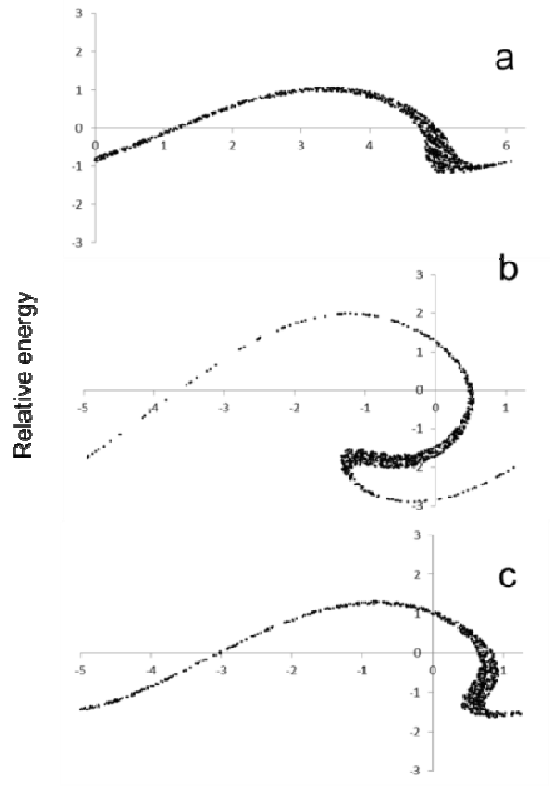

Phase [rad]

Figure 10: Phase distribution of the micro bunch for different momentum compaction: a $-R 56=0 \mu \mathrm{m}, L u=40 \mathrm{~m} ; \mathrm{b}-$ $R 56=-2 \mu \mathrm{m}, L u=40 \mathrm{~m} ; \mathrm{c}-R 56=-2 \mu \mathrm{m}, L u=35 \mathrm{~m}$.

For more flexibility and practical reasons, we can install three quadrupoles symmetrically within the chicane in order to allow a variation of the $R 56$ while maintaining the beam trajectory and cancellation of dispersion. Fig. 11 shows an example of $\pm 20 \%$ change of the momentum compaction by adjusting the quadrupole fields. The quadrupoles may, however, perturb the beta functions which can be compensated downstream of the chicane.

In the proposed scheme, the best X-ray beam will be generated by the last bunch in the train. The use of the X-ray beams generated by the other bunches will depend upon the users. If they would like to have only the best (last) X-ray beam, then all the other X-ray beams must be not generated. That means that the corresponding electron bunches must be slightly deflected after the downstream chicane in order to not generate X-rays in the undulators. This can be achieved in the same way that it is currently being used to measure the gain at the LCLS.

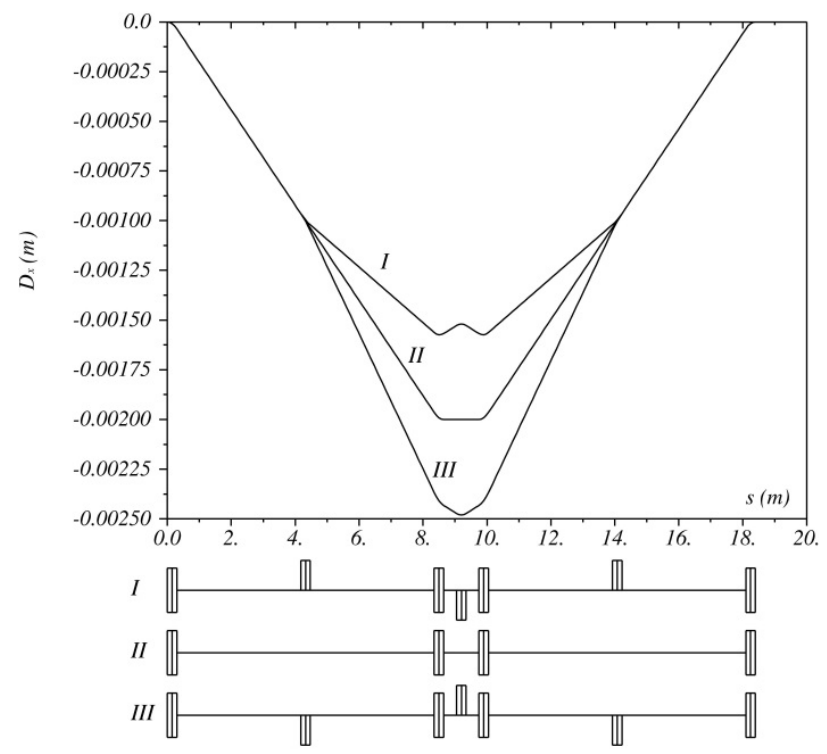

Figure 11: Variation of the dispersion functions in the chicane, corresponding to $R 56$ of $-0.8 \mu \mathrm{m}(I),-1.0 \mu \mathrm{m}(I I)$, and $1.2 \mu \mathrm{m}(I I I)$, using adjustment of the field in the 3 quadrupoles. In case $I I$, the quadrupoles are off. 


\section{CONCLUSION}

In the simulations we have achieved very efficient lasing with the regenerative amplifier free-electron laser. The results demonstrate the utility of an X-ray cavity to achieve high-monochromatic X-rays. Work is in progress to further understand and finalize the details of this proposal.

\section{ACKNOWLEDGEMENTS}

The authors would like to thank the LCLS physicists of the Accelerator Department for their help and fruitful discussions, especially Z. Huang, H.-D. Nuhn and A. Brachmann. This work was supported by Department of Energy Contract No. DOE-AC02-76SF00515.

\section{REFERENCES}

[1] J. Amann et al., "Demonstration of self-seeding in a hard-X-ray free-electron laser", Nature Photonics, Vol. 6, Oct. 2012, pp.693-698.

[2] J. Feldhaus, E. Saldin, J. Schneider, E. Schneidmiller, M. Yurkov, "Possible application of X-ray optical elements for reducing the spectral bandwidth of an X-ray SASE FEL”, Opt. Comm. 140, pp. 341-352 (1997).

[3] E. Saldin, E. Schneidmiller, Yu. Shvyd'ko, and M. Yurkov, "X-ray FEL with a meV bandwidth", Nucl. Instrum. Methods A 475, 357-362 (2001).

[4] A. Bhowmik, J. Madey and S. Benson, "Design concept for a common RF accelerator driven free electron laser master oscillator/power amplifier", Nucl. Instrum. Methods A 272, 183-186 (1988).

[5] L. Vintro et al, "Observation of Gain in a Free-Electron-Laser Master Oscillator-Power Amplifier”, PTL 64, 1662 (1990).

[6] F. Ciocci et al., "Design Considerations on a High-Power VUV FEL”, EEE JQE, VOL. 31, NO. 7, 1995

[7] K.-J. Kim, Yu. Shvyd'ko and S. Reicher, "A Proposal for an X-Ray Free-Electron Laser Oscillator with an EnergyRecovery Linac", PRL 100, 244802 (2008).

[8] D. C. Nguyen et al., "Initial results of the infrared regenerative amplifier FEL", in: Coherent Electron-beam X-ray Sources, Proceedings SPIE, vol. 3154, 1997, pp. 39-50.

[9] N. R. Thompson et.al, "A 3D model of the 4GLS VUV-FEL conceptual design including improved modelling of the optical cavity”, in Proceedings of FEL 2006, BESSY, Berlin, Germany, p. 304 (2006).

[10]Z. Huang, R. Ruth, "Fully Coherent X-Ray Pulses from a Regenerative-Amplifier Free-Electron Laser", PRL 96, 144801 (2006).

[11] A. Novokhatski, F.-J. Decker, B. Hettel, Z. Huang, H.-D. Nuhn and M. Sullivan, "Coherent X-ray Seeding Source for Driving FELS”, Proceedings of FEL2013, New York, NY, USA, p. 522, 2013.

[12] LCLS Conceptual Design Report No. SLAC-R-593, edited by J. Galayda, 2002.

[13] W. Roseker, H. Franz, H. Schulte-Schrepping, et al., Optics Letters, Vol. 34, No. 12, p. $1768,2009$.

[14] Kwang-Je Kim, “An Analysis of Self-Amplified Spontaneous Emission”, Nucl. Instrum. Methods A250, pp. 396403 (1986)

[15] R. Bonifacio and B.W.J. McNeil, "Slippage and superradiance in the high-gain FEL", Nucl. Instrum. Methods A 272, 280 (1988)

[16] Z. Huang, Private communications

[17] M. S. del Rio and R. Dejus, "XOP -X-ray Oriented Programs”.

[18] V. Lucarini et al., "Kramers-Kronig relations in optical materials research”, Springer, (2004).

[19] G. Geloni, V. Kocharyan and E. Saldin, "A simple method for controlling the line width of SASE X-ray FELs", DESY 10-053, April 2010.

[20] L. Bentson, P. Emma, "A new bunch compressor chicane for the SLAC linac to produce 39 fs, 30-kA, 30-GeV electron bunches", Proceedings of EPAC 2002, Paris, France, p. 683.

[21] H. Grote, F. Ch. Iselin, “The MAD Program (Methodical Acellerator Design), Version 8.19: User's Reference Manual”, CERN-SL-90-13-AP, Geneva, Switzerland (1996). 